\title{
A Neural Network Model for Predicting Epileptic Seizures based on Fourier-Bessel Functions
}

\author{
Shaik.Jakeer Husain ${ }^{1}$ and Dr.K.S.Rao ${ }^{2}$ \\ ${ }^{1}$ Associate professor, Dept. of Electronics and Communication Engineering, \\ Vidya Jyothi Institute of Technology, Hyderabad \\ ${ }^{2}$ Director, CVSR College of engineering, Hyderabad \\ jk.shaik@gmail.com
}

\begin{abstract}
To improve the social life of drug resistant epilepsy persons, a patient specific algorithm is needed that can predict seizures based on EEG with high sensitivity and specificity before the occurrence of a seizure. This algorithm predicting the seizure occurrence from Inter-ictal (seizure free) and pre-ictal (before seizure) transition. In this algorithm features are extracted by Fourier Bessel Expansion from inter-ictal and pre-ictal EEG signals. A neural network using back propagation algorithm is implemented for classification of epileptic states. The performance of algorithm is evaluated based on three measures, sensitivity, and specificity and classification accuracy. The results illustrate that the algorithm can predict seizures of two subjects before five minutes with an accuracy of $99.6 \%$
\end{abstract}

Keywords: EEG signals, Epileptic seizure prediction, Fourier Bessel Coefficients (FBC), Artificial Neural Networks (ANN).

\section{Introduction}

Even if epileptic seizures are rare in a given patient, the constant fear of the next seizure and the feeling of helplessness often have a strong impact on the daily life of a patient. A method reliably predicts the occurrence of seizures could significantly improve the quality of life for these patients, and open new therapeutic possibilities such as on demand drug delivery or on demand electrical stimulation which resets brain dynamics. It has long been observed that the transition from the inter-ictal state (far from seizures) to the ictal state (seizure) is not sudden and maybe preceded from minutes to hours by clinical, metabolic or electrical changes. The goal of seizure prediction problem is to predict an upcoming seizure based on the analysis of biomedical signal recorded from patients. In seizure prediction problems, there are some basic terms as follow:

1) The ictal state is a period of time in which seizure onset is identified by epileptologists through EEG wave-form examination.

2) The preictal state is a period of time before the seizure onset occurs.

3) The postictal state is a period of time after the seizure onset ends.

4) The interictal state is other than the above three states.

Note that in seizure prediction problem, the duration of each state is decided by human speculation rather than an objective value since the true mechanisms of spontaneous occurrence of seizures are not completely understood. Generally, the data corresponding to ictal and post-ictal is discarded in this setting, because the task is to predict a upcoming seizure Prediction [1-5, 9-10]. Seizure prediction approaches can be summarized into two steps. The first is extracting Features from EEG over time. The second is classifying them 
into a Pre-ictal or Inter-ictal state using statistical analysis or other machine learning techniques such as neural network and support vector machine. Pattern recognition techniques relay on the ability to generate a set of coefficients from the raw data (time domain samples) that are more compact and more closely related to the signal characteristics of interest [7]. A compact representation of EEG signal is possible using Bessel functions because of the similarity between EEG signals and the Bessel functions. [8 - 11]

In this paper we are interested in expanding an EEG signal into a Fourier-Bessel series. The coefficients of the Fourier-Bessel (FB) series expansion have been used to constitute a feature vector for segmentation of the EEG signal. These coefficients are used to classifying inter-ictal and pre-ictal to predict the seizure before its occurrence.

\section{Methodology}

\section{FOURIER BESSEL EXPANSION}

The Fourier Bessel series is expressed according to the mathematical equation:

$$
x(n)=\sum_{m=1}^{M} C_{m} J_{0}\left(\lambda_{m} \frac{n}{N}\right)
$$

Where $C_{m}$ is the $m^{\text {th }}$ Fourier Bessel coefficient which can be expressed as [3]

$$
C_{m}=\frac{2 \sum_{n=0}^{N} n x(n) J_{0}\left(\frac{\lambda_{n}}{N}\right)}{N^{2}\left(J_{0}\left(\lambda_{m}\right)\right)^{2}}
$$

Where $J_{0}$ is zero order Bessel Function of the first kind and $\lambda_{n}$ is its $n^{\text {th }}$ root and $\mathrm{N}$ is the length of the time series. With a given number of $C_{m}$ `s we can reconstruct the signal. The paper attempts to model the mono-components intrinsic to the signal by reconstructing only from a specified number of $C_{m}$ `s at a time. There exists a one to one mapping from the frequencies inherit to the signal and Fourier-Bessel coefficients.

$$
\frac{f}{\left(\frac{f_{s}}{2}\right)}=\frac{m}{n}
$$

Where is the $f_{s}$ sampling frequency Unlike Fourier series where the signal needs to be periodic the decaying nature of the Fourier-Bessel allows us to model any signal. $f_{s}=256$ and $n=128$ (number of Fourier Bessel Coefficients)

Table 1. One to One Mapping of Frequencies to the Fourier-Bessel Coefficients

\begin{tabular}{|l|l|l|}
\hline EEG Sub Band & Frequency & Fourier-Bessel \\
& Range $(\mathrm{Hz})$ & Coefficient(m) \\
\hline DELTA & $0-4$ & $0-4$ \\
\hline THEETA & $4-7$ & $4-7$ \\
\hline ALPHA & $7-13$ & $7-13$ \\
\hline LOW BETA & $13-15$ & $13-15$ \\
\hline
\end{tabular}




\begin{tabular}{|l|l|l|}
\hline HIGH BETA & $15-30$ & $15-30$ \\
\hline LOW GAMA & $30-65$ & $30-65$ \\
\hline HIGH GAMA & $65-120$ & $65-120$ \\
\hline
\end{tabular}
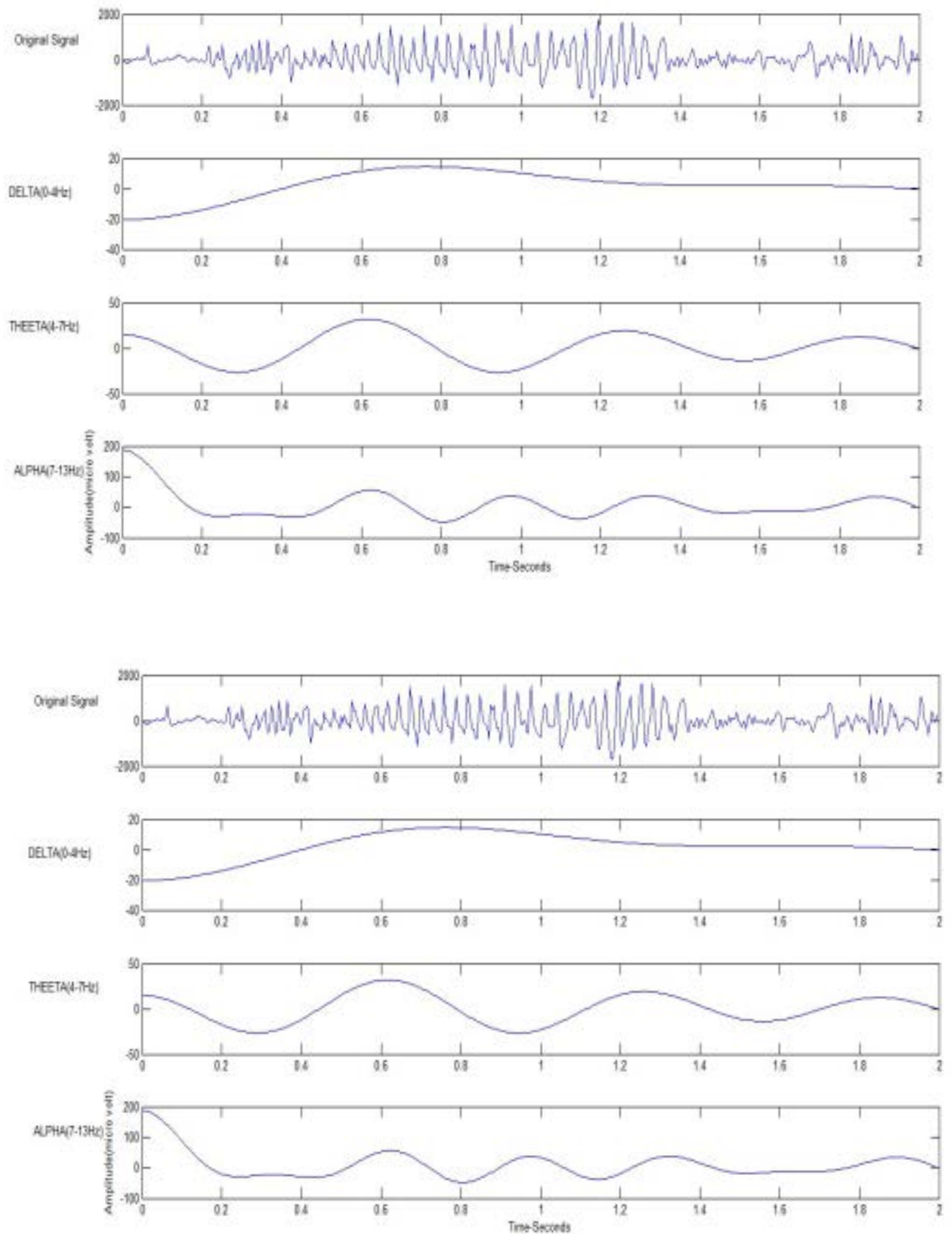

Figure 1. Segmented EEG signal --Seizure (Ictal)

Table I shows That Fourier-Bessel coefficients corresponding to each sub band. Figure 1 shows the segmentation of seizure signal. The signal is segmented into seven bands Delta, 
Theta, Alpha, Low Beta, High Beta, Low Gamma and High Gamma. Figure 2 shows the Inter -ictal signal (seizure free) and its seven segmented bands. Figure 3 shows preictal signal (5 min before seizure) and segmented bands.

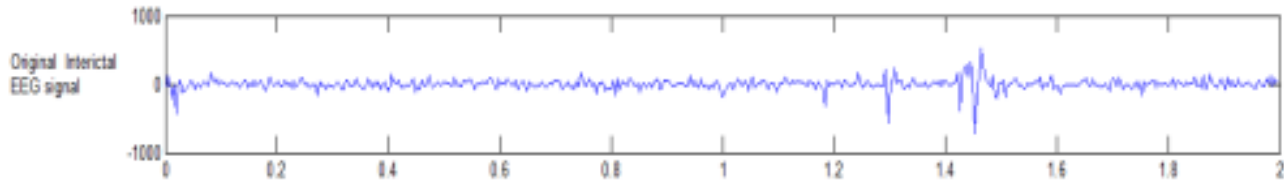

DEITA
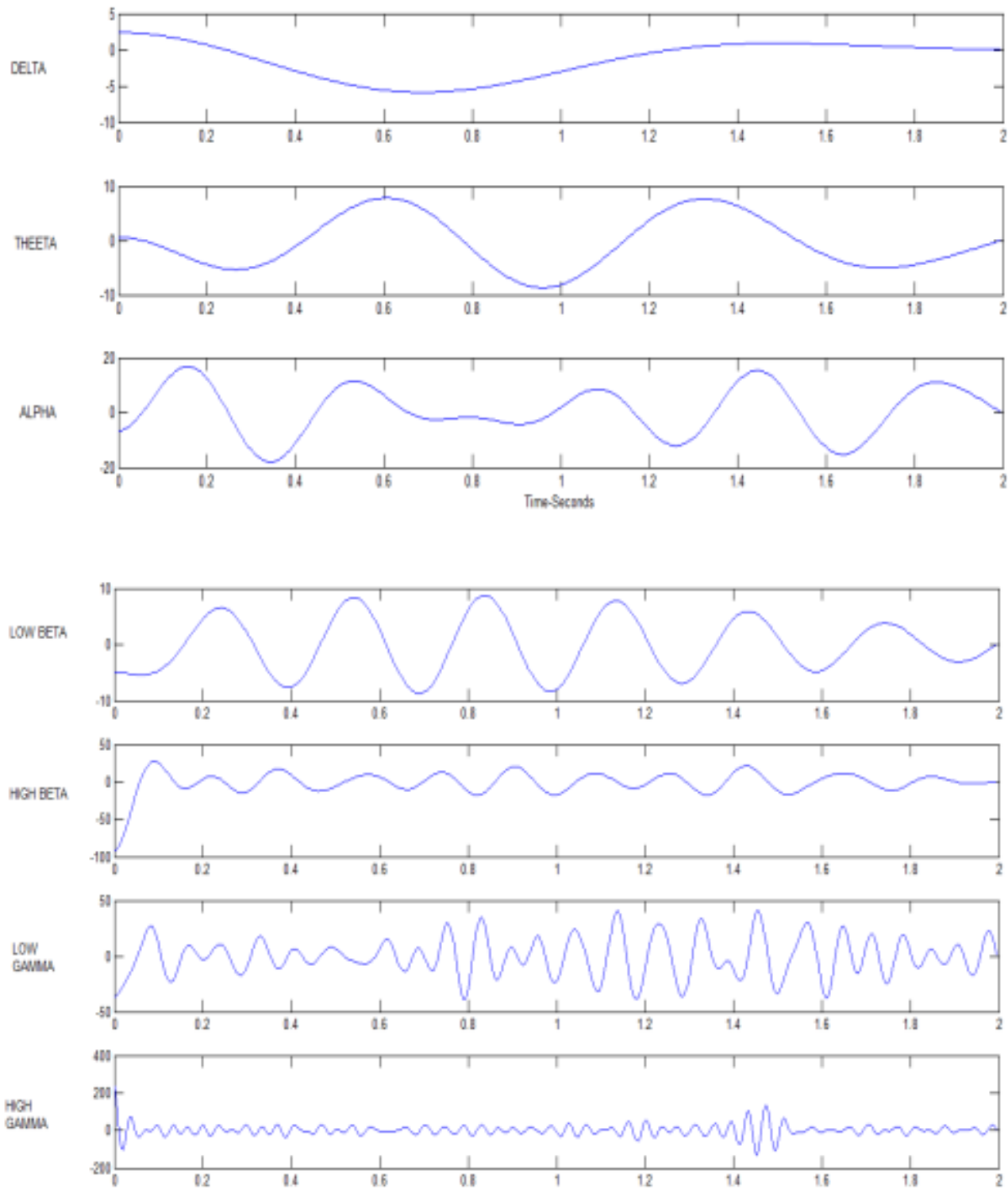

Figure 2. Segmented EEG signal --Inter-ictal 

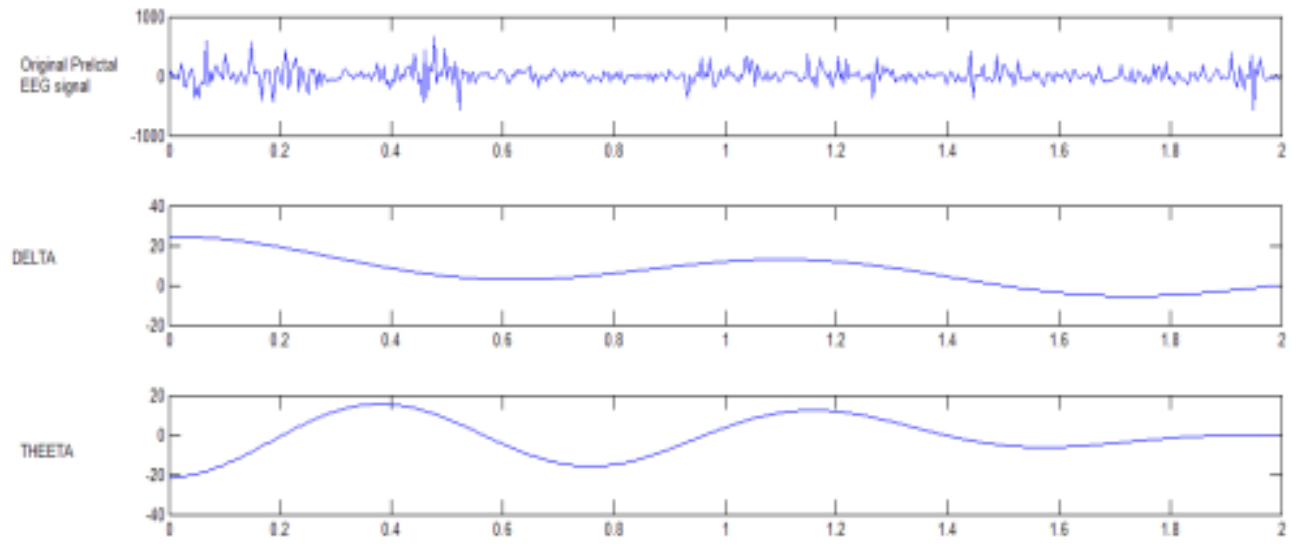

NPOHA

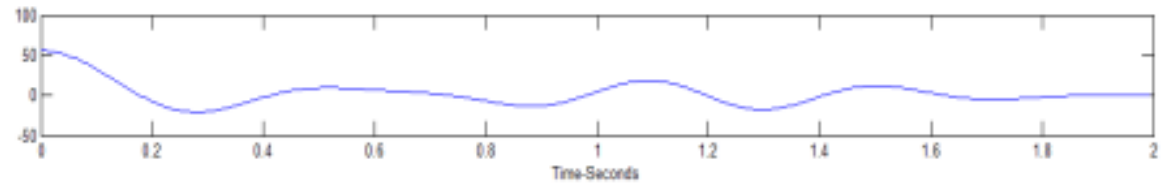

LOW EETA

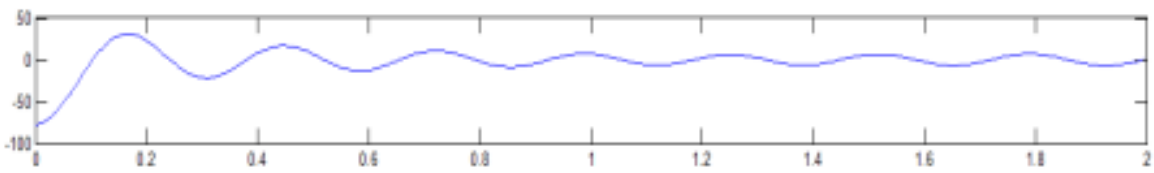

HISH BETA

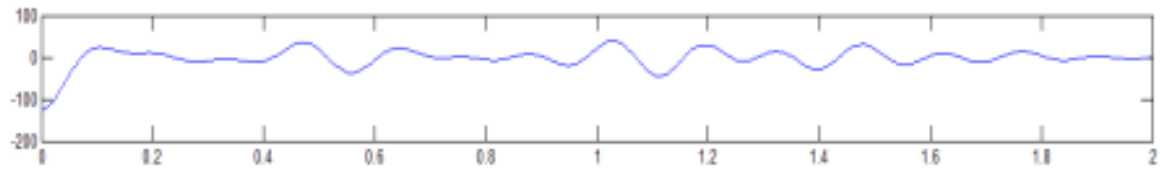

LOW GAMu
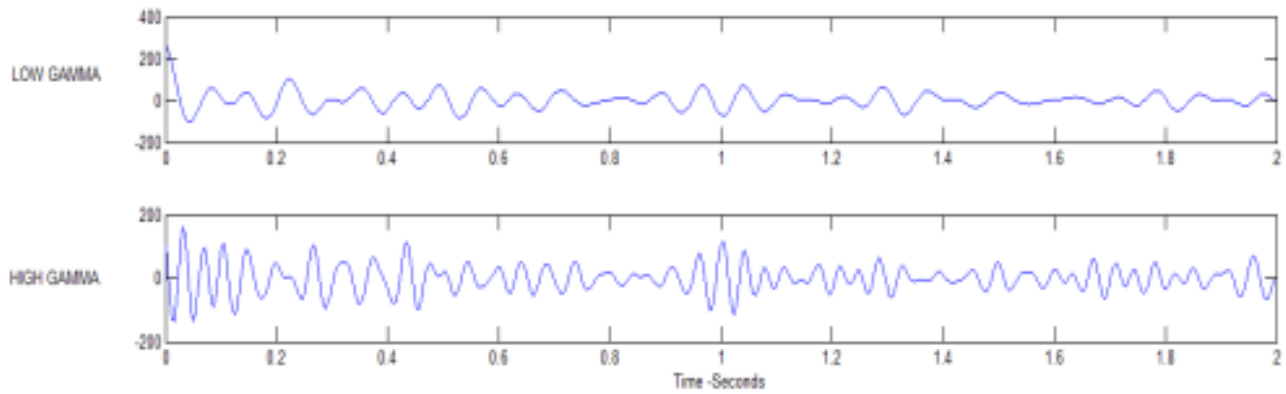

Figure 3. Segmented EEG signal --Pre-ictal

\subsection{Feature Extraction}

Following Five features are extracted from each sub-band

I. An Energy Measure From Each Sub band-passed

$$
e_{k}=\sum_{i=m 1}^{m 2}\left|C_{i}\right|
$$

Where C is Fourier Bessel coefficient and $\mathrm{k}$ is sub band (In Table-1 mentioned corresponding coefficients to each band) 
II. Energy in each sub band

$$
E_{k}=\sum_{i=m 1}^{m 2} 2 C^{2}{ }_{k} \frac{N^{2}}{2} J_{1}\left(\lambda_{k}\right)^{2}
$$

III $\mathrm{f}_{\text {mean is }}$ calculated using $\mathrm{E}_{\mathrm{k}}$ and $\mathrm{f}_{\mathrm{k}}$

$$
f_{\text {mean }}=\frac{\sum_{i=m 1}^{m 2} f_{k} E_{k}}{\sum_{i=m 1}^{m 2} E_{k}}
$$

IV Inter quartile range in each band IQR is defined by

$$
I Q R=Q_{3}-Q_{1}
$$

Where, Q1 and Q3 are the first and third quartile respectively. [11]

$\mathrm{V}$ Median absolute deviation in each band. The median absolute deviation is the mean of the absolute deviations of a set of data about the data's mean. For a sample size $N$ and the mean distribution $\mathrm{x}$, the median absolute deviation is defined by (MAD) [11]

$$
\frac{1}{N} \sum_{i=1}^{N}\left|x_{i}-\bar{x}\right|
$$

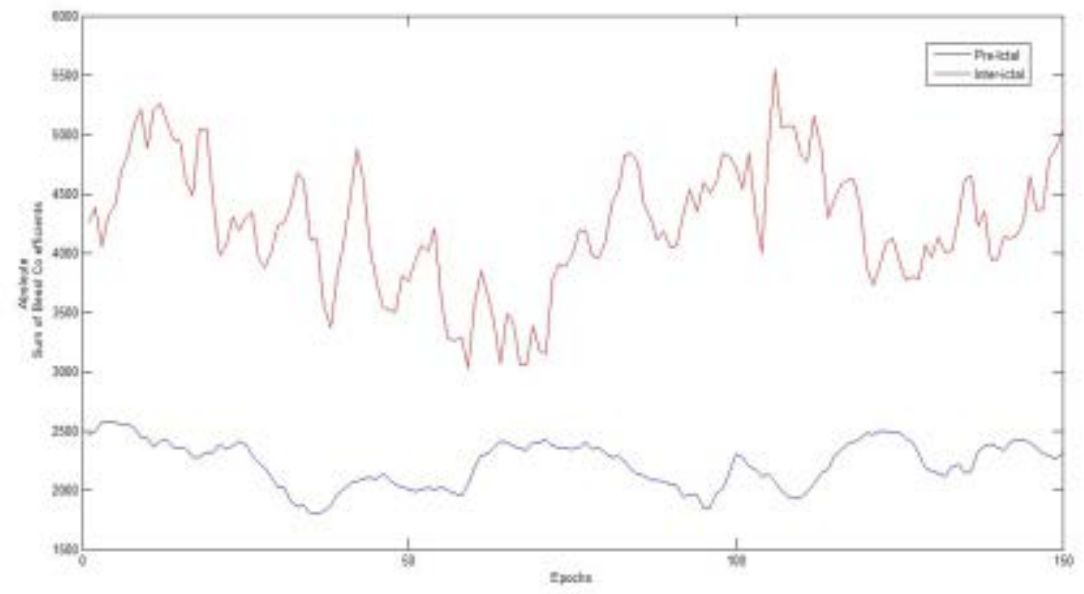

\section{Figure 4. Absolute Sum of Bessel Coefficients (Preictal and Interictal EEG Signals)}

The five features discussed in 2.1 are extracted from each sub band. The Figure 4 shows the sum of all Bessel coefficients the preictal and interictal features are discriminating. The Figure 5 shows the Median absolute deviation of Fourier Bessel coefficients, Intrictal and preictal features are discriminating We found that the feature values of two lower bands remain relatively constant, we can select only five higher bands: alpha $(7-13 \mathrm{~Hz})$, low beta (13-15 Hz), high beta $(15-30 \mathrm{~Hz})$, low gamma (30-65 Hz), and high gamma $(65-120 \mathrm{~Hz})$ instead of seven bands. Fourier Bessel coefficients are unique for a given signal, to reduce the computational load coefficients are directly used for classification 


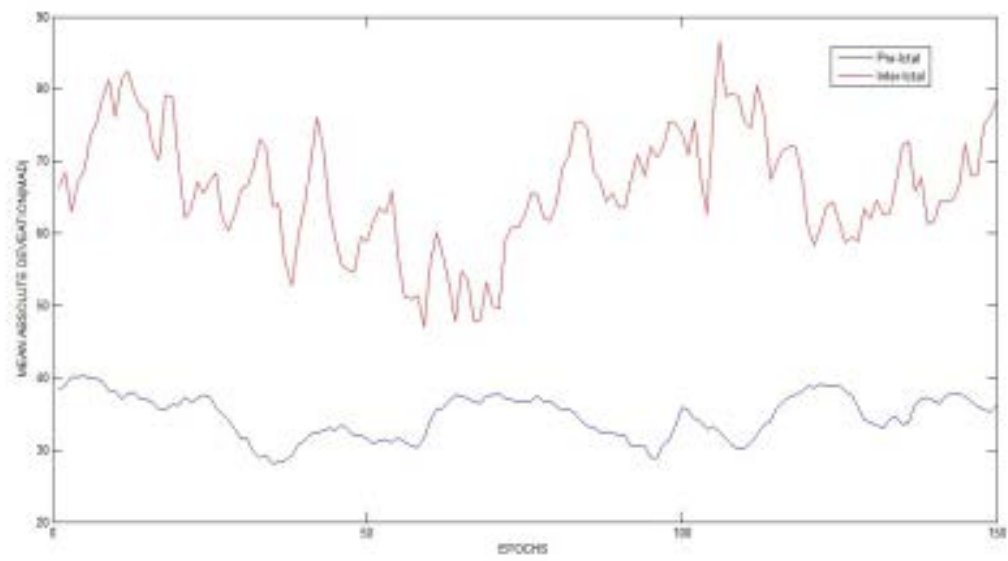

Figure 5. Median Absolute Deviation of Coefficients (Preictal and Interictal EEG Signals)

\section{Experimental Results and Discussion}

The database, used is collected from the Children's Hospital Boston, consists of EEG recordings from pediatric subjects with intractable seizures. All signals were sampled at 256 samples per second [5].

Table 2. Subject-1 Information

\begin{tabular}{|l|l|l|c|l|l|}
\hline \multicolumn{1}{|c|}{ File } & \multicolumn{1}{|c|}{ File } & File & Number of & Seizure Start & Seizure End \\
& Start Time & End Time & Seizures & seconds & seconds \\
\hline chb01_01 & $11: 42: 54$ & $12: 42: 54$ & 0 & -- & -- \\
\hline chb01_03 & $13: 43: 04$ & $14: 43: 04$ & 1 & 2996 & 3036 \\
\hline chb01_15 & $01: 44: 44$ & $2: 44: 44$ & 1 & 1732 & 1772 \\
\hline
\end{tabular}

The inter-ictal and pre -ictal data is prepared as per the information in Table 2. The interictal data selected such that more than 30 minute no seizure occurred before and after the inter ictal data. The pre-ictal data is 5 minutes before the seizure. From 512 (2 seconds Epoch) samples 64 Fourier-Bessel coefficients are calculated these coefficients are considered as Feature vector. The calculated Fourier-Bessel Coefficients from inter ictal and pre ictal data is given to Neural Network with 64 input neurons, one output neuron and one hidden layer. We used the Feed Forward Back propagation algorithm shows in Figure 6. The network is trained -1 as target for inter-ictal and +1 for pre-ictal. 


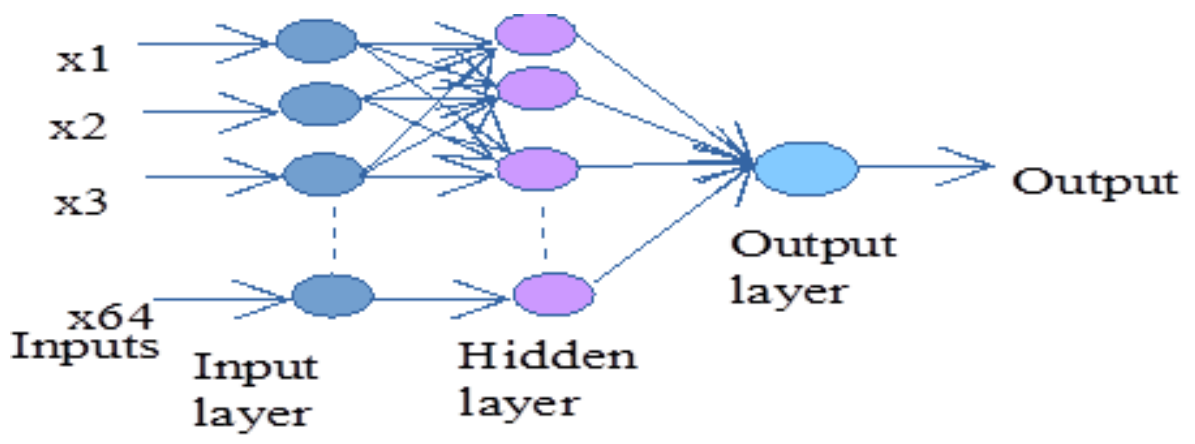

Figure 6. Neural Network Architecture

The trained network is simulated with Inter-ictal and Pre-ictal data. We got one epoch as false negative and zero false positives. Figure 7 shows the simulation results of 150 epochs of inter -ictal and 150 epochs of pre-ictal data.

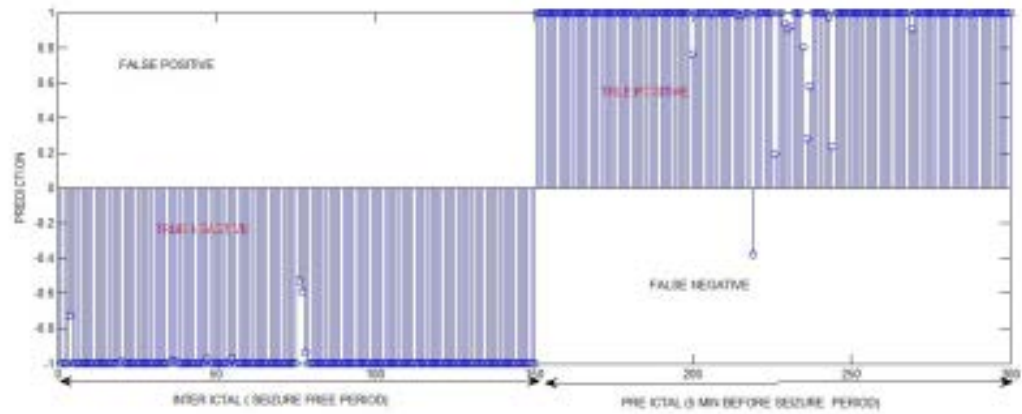

Figure 7. Subject-1 Prediction Simulation

Sensitivity (SE): number of true positive(TP) decisions / number of actually positive cases. Specificity (SP): number of true negative (TN) decisions / number of actually negative cases. Total classification accuracy (TCA): number of correct decisions / total number of cases.

Table 3. Sensitivity, Specificity and Classification Accuracy

\begin{tabular}{|l|l|l|l|l|l|l|}
\hline TP & FN & SE & TN & FP & SP & TCA \\
\hline 149 & 01 & 99.33 & 150 & 0 & $100 \%$ & $99.6 \%$ \\
\hline
\end{tabular}

From TABLE 3, it is observed that sensitivity, specificity and accuracy of the proposed method is superior and the seizure is predicted before 5 minutes for subject 1 .

Table 4. Subject 2 Information

\begin{tabular}{|l|l|l|l|}
\hline \multicolumn{1}{|c|}{ File Name } & Number of Seizures & Seizure Start(seconds) & Seizure End(seconds) \\
\hline chb24_13 & 1 & 3288 & 3304 \\
\hline chb24_14 & 1 & 1939 & 1966 \\
\hline chb24_15 & 1 & 3552 & 3569 \\
& & & \\
\hline
\end{tabular}


The inter-ictal and pre ictal data is prepared as per the information in Table 4. The calculated Fourier-Bessel Coefficients from inter-ictal and pre-ictal data is given to Neural Network with 64 input neurons, one output neuron and one hidden layer. We used the Feed Forward Back propagation algorithm shows in Figure 6. The network is trained 0 as target for inter-ictal and +1 for prei-ctal.

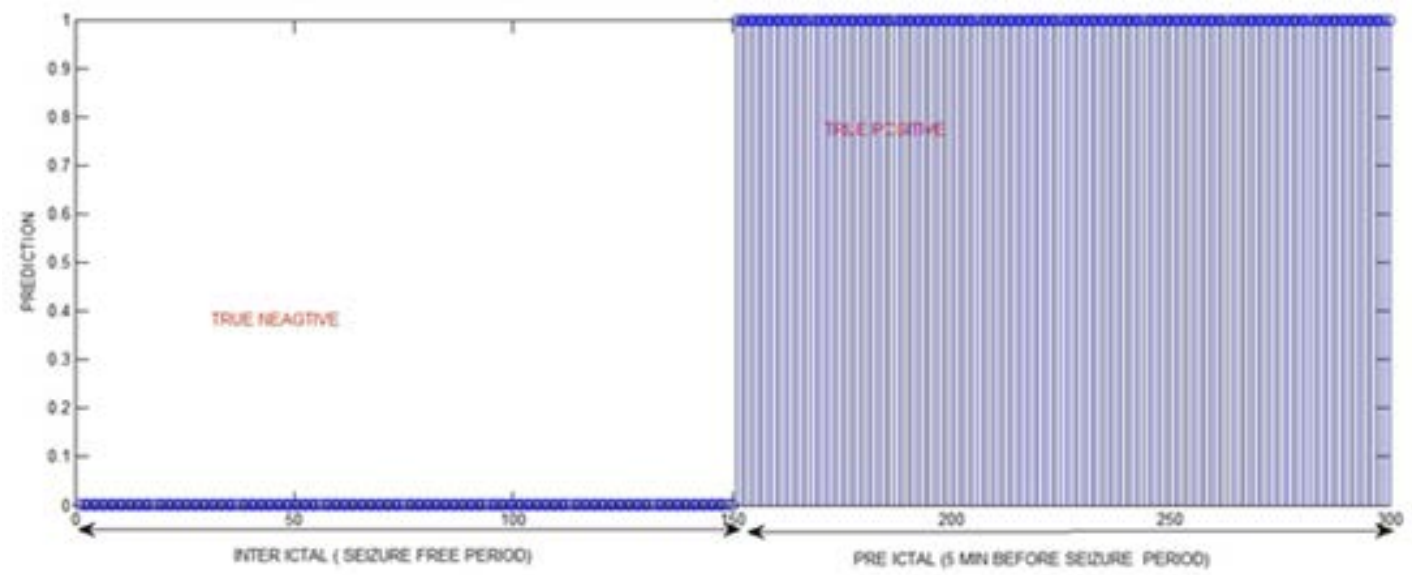

Figure 8. Subject-2 Prediction Simulation

The trained network is simulated with inter-ictal and pre-ictal data. We got one epoch as false negative and zero false positives. Figure 8 shows the simulation results of 150 epochs of inter-ictal and 150 epochs of pre-ictal data.

Table 5. Sensitivity, Specificity and Classification Accuracy

\begin{tabular}{|l|l|l|l|l|l|l|}
\hline TP & FN & SE & TN & FP & SP & TCA \\
\hline 150 & 0 & $100 \%$ & 150 & 0 & $100 \%$ & $100 \%$ \\
\hline
\end{tabular}

From TABLE 5 it is observed that s seizure is predicted before 5 minutes for subject 2 with $100 \%$ accuracy

\section{Conclusions}

In this paper, we have presented a new patient-specific system for the prediction of epileptic seizures from the inter-ictal and pre-ictal EEG. Features are extracted using Fourier Bessel expansion. The extracted features are classified using neural network. The results are shown in Table 3 and Table 5 as it can be seen, the proposed prediction method has the best results.

There is a still issue in seizure prediction problem that has not been properly resolved, which is variation issue [5]. A model trained from several seizures may have no predictive power for the upcoming seizures in next few days or even few hours. It is too optimistic to expect the performance of the trained model to persist while the patient condition varies constantly. Also, the amount of past seizures that a trained model needs to learn remains a basic issue in seizure prediction problem. On the other hand, it is also important to reduce false alarms to relieve patients from being worried about nonexistent upcoming seizures. This can resolve by incorporating more recent data into classifier retraining. Further, the proposed method leads to a promising foundation for future seizure prediction method development. 


\section{References}

[1] B. Litt and K. Lehnertz, "Seizure prediction and the pre seizure period", Current Opinion in Neurology, vol. 15, (2002), pp. 173-177.

[2] B. Litt and J. Echauz, "Prediction of epileptic seizures”, The Lancet Neurology, vol. 1, (2002) May, pp. 2230.

[3] L. D. Iasemidis, D. S. Shiau, W. Chaovalitwongse, J. Sackellares, P. M. Pardalos, J. C. Principe, P. R. Carney, A. Prasad, B. Veeramani and K. T. Sakalis, “Adaptive epileptic seizure prediction system”, IEEE Trans. Biomed. Eng., vol. 50, (2003), pp. 616-627.

[4] A. Schad, K. Schindler, B. Schelter, T. Maiwald, A. Brandt and J. Timmer, "Application of a multivariate seizure detection and prediction method to non-invasive and intracranial long-term EEG recordings”, Clinical Neurophysiology, vol. 119, (2008), pp. 197-211.

[5] C.-Y. Chiang, N. F. Chang, T. C. Chen, H.-H. Chen and L. G. Chen, "Seizure Prediction Based on Classification of EEG Synchronisation Patterns with On line Retraining and Post-Processing Scheme”, Proceeding of $33^{\text {rd }}$ Annual International Conférence of the IEEE EMBS Boston, (2011) August 30, Massachusetts USA.

[6] R. P. Costa, P. Oliveira, G. Rodrigues, O. B. Leita and A. Dourado, "Epileptic seizure classification using neural networks with 14 features”, (2008), pp. 281-288.

[7] J. Schroeder, "Signal processing via Fourier-Bessel series expansion”, Digital Signal Processing, vol. 3, (1993), pp. 112-124.

[8] R. B. Pachori and P. Sircar, "EEG signal analysis using FB expansion and second-order linear TVAR process”, Signal Processing, vol. 88, no. 2, (2008), pp. 415-420.

[9] P. W. Mirowski, Y. L. Cun, D. Madhavan and R. Kuzniecky, "Comparing SVM and Convolutional Networks for Epileptic Seizure Prediction from Intracranial EEG”, IEEE, (2008), pp. 244-249.

[10] A. S. Zandi, R. T. Hi, M. J. Avidan and G. A Dumont, "Predicting temporal lobe epileptic seizures based on zero-crossing interval analysis is in scalp EEG,” Proceeding of, 32nd Annual International IEEE EMBS Conference, (2010), Buenos Ares, Argentina.

[11] R. Shaik. J. Husain and K. S. Rao, "Epileptic Seizures Classification from EEG Signals using Neural Networks”, Networks Proceeding of International Conference on Information and Network Technology ICINT, (2012).

\section{Authors}

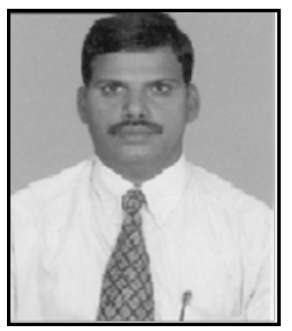

Shaik Jakeer Husain, he received the B.E degree in Electronics and Communication Engineering from Andhra University, Visakhapatnam in 1996, and M.E in Digital System from Osmania University, Hyderabad in 2008 and he is pursuing PhD in Digital Signal Processing at JNTU, Hyderabad. He is currently an Associate Professor in Department of Electronics and Communication Engineering at Vidya Jyothi Institute of Technology, Hyderabad. His research interests include Biomedical Signal Processing and Digital Signal Processing

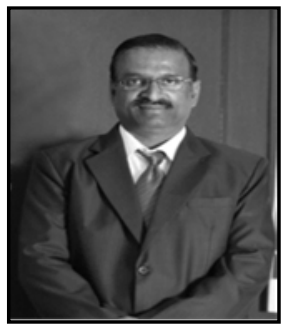

K. S. Rao, he obtained his B. Tech, M. Tech and Ph.D. in Electronics and Instrumentation Engineering in the years 1986, 89 and 97 from KITS, REC Warangal and VRCE Nagpur respectively. He had 25 years of teaching and research experience and worked in all academic positions, presently he is the Director, Anurag Group of Institutions (Autonomous) Hyderabad. His fields of interests are Signal Processing, Neural Networks and VLSI system design. 